\title{
The study of entropy in a transmission line resonator interacting with a capacitively coupled Cooper pair box
}

\author{
Clodoaldo Valverde ${ }^{* 1,2}$ and Basílio Baseia ${ }^{3,4}$ \\ ${ }^{1}$ Laboratório de Modelagem Molecular Aplicada e Simulação (LaMMAS), Universidade Estadual de Goiás, 75001 - \\ 970, Anápolis, GO, Brazil, \\ ${ }^{2}$ Universidade Paulista, 74845-090, Goiânia, GO, Brazil, \\ ${ }^{3}$ Departamento de Física, Universidade Federal da Paraíba, 58.051-970, João Pessoa, PB, Brazil, \\ ${ }^{4}$ Instituto de Física, Universidade Federal de Goiás, 74.690-900, Goiânia, GO, Brazil.
}

Received July 27, 2019; accepted September 26, 2019; published September 30, 2019

\begin{abstract}
In the present work, we theoretically study the entropy of a Cooper pair box coupled to a (single mode) of onedimensional transmission line resonator. The proposed scheme uses the Jaynes-Cummings model in presence of losses to study the time evolution of the system entropy. The coupling of these two subsystems provides an important indication of the influence of a Cooper pair box on the evolution of system entropy. Decaying CPB allows us to control the variation of entropy at certain times.
\end{abstract}

Hybrid systems in the treatment of quantum information have gained great interest in recent years [1-2], due to the great advantage of combining atoms, spins and solid state devices with various applications, for example quantum computation and quantum information [3-4], quantum state engineering [5-8], atomic physics and quantum optics [9-10], photon blockade [11-12], quantum dynamics [13], and propagating phonons [14]. Hybrid systems besides being an imminently robust architecture open a new frontier for studying the ultra-strong coupling between individual microwave photons and "atoms" [15]. It is also possible to build hybrid quantum devices [16] that combine infinite degrees of freedom from different physical systems. In addition, they provide an alternative path for quantum mechanics testing under an unattainable size and mass parameter scheme [17-20]. In this work we have employed the Jaynes-Cummings (JC) model to treat the Cooper-pair box (CPB) coupled to a single mode of one-dimensional transmission line resonator (TLR) [20$21]$ in the presence of losses [23] and the action of a timedependent external field.

The TLR-CPB hybrid system is presented in Fig. 1.

*E-mail: valverde@ueg.br

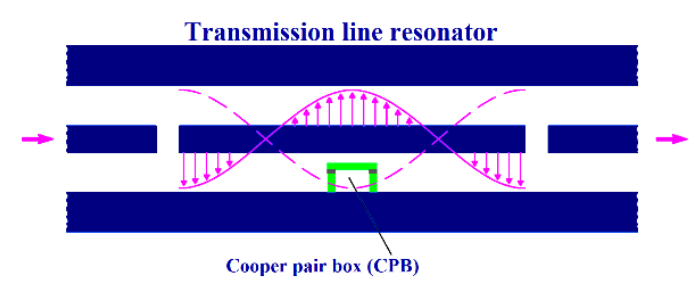

Fig. 1. Schematic of the arrangement to investigate the system.

We investigated the evolution of the entropy of the TLRCPB hybrid system. To discuss the requirements of the present proposal we will consider the system in the presence of losses and, therefore, including decoherence effects; to this end, let us take the following Hamiltonian,

$\widehat{H}=\widehat{H}_{T L R}+\widehat{H}_{C P B}+\widehat{H}_{J C}+\widehat{H}_{D}$,

where $\widehat{H}_{T L R}$ reports the transmission line resonator, $\widehat{H}_{C P B}$ describes the CPB subsystem, $\widehat{H}_{J C}$ represents the coupling between TLR-CPB and $\widehat{H}_{D}$ symbolizes the CPB losses. By explicitly rewriting Eq. (1) we have,

$$
\begin{gathered}
\widehat{H}=\omega \hat{a}^{\dagger} \hat{a}+\frac{1}{2} \omega_{C P B} \hat{\sigma}_{z}+\delta\left(\hat{\sigma}_{+} \hat{a}+\hat{a}^{\dagger} \hat{\sigma}_{-}\right) \\
-i \frac{\beta}{2}|1\rangle\langle 1|,
\end{gathered}
$$

where $\omega$ is the frequency of TLR and $\hat{a}\left(\hat{a}^{\dagger}\right)$ stands for the annihilation (creation) operator of the field (TRL); $\hat{\sigma}_{-}\left(\hat{\sigma}_{+}\right)$is the lowering (raising) operator acting on the $\mathrm{CPB}, \hat{\sigma}_{z}$ is the Pauli operator $\left(\hat{\sigma}_{z}=|1\rangle\langle 1|-| 0\rangle\langle 0|\right)$, $\omega_{C P B}$ is the CPB frequency, and $\delta$ stands for theTLR$\mathrm{CPB}$ coupling strength. The wave function that describes the time evolution of the whole TLR-CPB system can be written as

$|\psi(t)\rangle=\sum_{n}^{\infty}\left[\varphi_{0, n}(t)|0, n\rangle+\varphi_{1, n}(t)|1, n\rangle\right]$,

where $|1\rangle(|0\rangle)$ represents the CPB in its excited (ground) state, $\mathrm{n}$ stands for the number of photons in the TLR, and 
$\varphi_{0, n}(t)$ and $\varphi_{1, n}(t)$ stand respectively for probability amplitudes of the states $|0, n\rangle$ and $|1, n\rangle$.

In the present context we will consider initially the CPB subsystem in its excited state and TLR subsystem in a coherent state $|\alpha\rangle$, the entire system being written as, $|\psi(0)\rangle=|1\rangle|\alpha\rangle$, where $|\alpha\rangle=\sum_{n=0}^{\infty} \exp \left(-\frac{|\alpha|^{2}}{2}\right) \frac{\alpha^{n}}{\sqrt{n !}}|n\rangle$.

As usual, we also assume that the TLR and CPB are initially decoupled, $\varphi_{0, n}(0)=0$ and $\sum_{n=0}^{\infty}\left|\varphi_{1, n}(0)\right|^{2}=$ 1 , thus $|\psi(0)\rangle=\sum_{n=0}^{\infty} \varphi_{1, n}(0)|1, n\rangle$.

The evolution of the time-dependent Schrodinger equation can be described as $(\hbar=1)$,

$i \frac{\partial|\psi(t)\rangle}{\partial t}=\widehat{H}|\psi(t)\rangle$,

where $\widehat{H}$ is the Hamiltonian of Eq. (2). Consequently, we can get a set of equations of motion expressed in the following,

$$
\begin{aligned}
\frac{\partial \varphi_{1, n}(t)}{\partial t}=-i n & \omega \varphi_{1, n}(t)-\frac{i}{2} \omega_{C P B} \varphi_{1, n}(t) \\
& -i \delta \sqrt{n+1} \varphi_{0, n+1}(t) \\
& -\frac{\beta}{2} \varphi_{1, n}(t)
\end{aligned}
$$

and

$$
\begin{aligned}
\frac{\partial \varphi_{0, n+1}(t)}{\partial t}=-i & (n+1) \omega \varphi_{0, n+1}(t) \\
& +\frac{i}{2} \omega_{C P B} \varphi_{0, n+1}(t) \\
& -i \delta \sqrt{n+1} \varphi_{1, n}(t)
\end{aligned}
$$

The solution of this system was solved numerically using the $\left(4^{\text {th }}\right.$ order $)$ Runge-Kutta method.

The effect concerning the von Neumann's entropy offers a quantitative measure of the disorder of a system as well as its degree of impurity, as shown by Phoenix and Knight [24]. This kind of entropy, determined in the form $S_{T L R-C P B}=-\operatorname{Tr}\left(\hat{\rho}_{T L R-C P B} \ln \left(\hat{\rho}_{T L R-C P B}\right)\right)$, is a measure of the mixing of two (or more) subsystems. The density operator $\hat{\rho}_{T L R-C P B}$ describes the entire system and can be defined as $\hat{\rho}_{T L R-C P B}=|\psi(t)\rangle\langle\psi(t)|$; so the entropy takes the form,

$$
\begin{array}{r}
S_{T L R-C P B}=-\left[N_{T C}^{+}(t) \ln \left(N_{T C}^{+}(t)\right)\right. \\
\left.+N_{T C}^{-}(t) \ln \left(N_{T C}^{-}(t)\right)\right],
\end{array}
$$

where the index TC is an abbreviation for $T L R-C P B$ and,

$$
\begin{aligned}
& N_{T C}^{ \pm}(t)=\frac{1}{2}\left[\left(\sum_{n=0}^{\infty}\left|\varphi_{1, n}(t)\right|^{2}+\sum_{n=0}^{\infty}\left|\varphi_{0, n+1}(t)\right|^{2}\right) \pm\right. \\
& \sqrt{\left(\begin{array}{c}
\left.\sum_{n=0}^{\infty}\left|\varphi_{1, n}(t)\right|^{2}-\sum_{n=0}^{\infty}\left|\varphi_{0, n+1}(t)\right|^{2}\right)^{2} \\
+4\left(\left|\sum_{n=0}^{\infty} \varphi_{1, n+1}^{*}(t) \varphi_{0, n+1}(t)\right|^{2}\right)
\end{array}\right]}
\end{aligned}
$$

The entropy $S_{T L R-C P B}$ is zero when $\hat{\rho}_{T L R-C P B}$ represents a pure state and is maximum and equal to $\ln (N)$ for a state of maximum mixing, where $N$ is the dimension of the Hilbert space. Nonetheless, here our state is pure only at $t=0$; for $t>0$ the state of the whole system loses its purity due to the action of time-dependent external fields and losses.

The results obtained are shown in Figs. 2 and 3. Figure 2 (a) concerns the lossless case in which entropy has an almost periodic character. After the beginning of the interaction, the TLR entropy tends to its minimum, then returns to its maximum and remains oscillating regularly due to the sequence of energy exchanges between the TLR and CPB subsystems. The entropy increases with the inclusion of CPB loss, in the interval $(25<\delta \mathrm{t}<50)$, see Fig. 2(b); this increase becomes smaller with time. When the loss in CPB increases the maximum value of entropy diminishes in the interval $(25<\delta \mathrm{t}<50)$, see Fig. 2 (c).
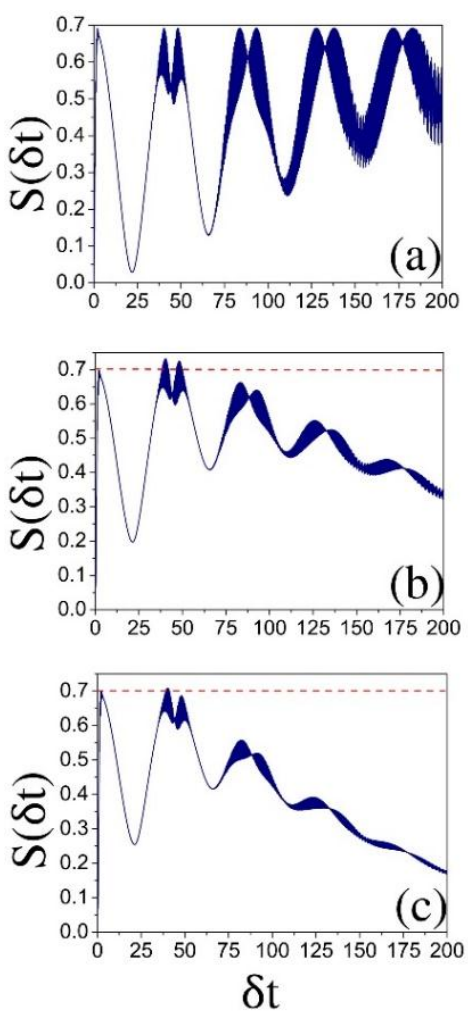

Fig. 2. Time evolution of the entropy when the TLR is initially prepared in a coherent state, for different values of the decay rate $\beta$ : (a) $\beta=0.0 \delta$, (b) $\beta=0.02 \delta$, and (c) $\beta=0.03 \delta$, with $\alpha=7, \omega=\omega_{\mathrm{CPB}}=8.35 \delta$.

Comparing Fig. 3(a) with Fig. 3(c) we note that entropy loses its periodic oscillations over time; a decrease in the minimum entropy amplitude is also observed. Both effects are due to losses on CPB. The time evolution of the TLR-CPB system shows a periodic increase and decrease of coherence as the system mixing increases. If the losses increase sufficiently, the values of maximum entropy of both subsystems go to zero. The TLR and CPB 
subsystems starting as pure (distinct) states end in vacuum states.

In this work we show that it is possible to control the entropy only via the control of the loss parameter $\beta$ in the $\mathrm{CPB}$; but this is only possible when this parameter varies in the range of $(0<\beta<0.02 \delta)$.
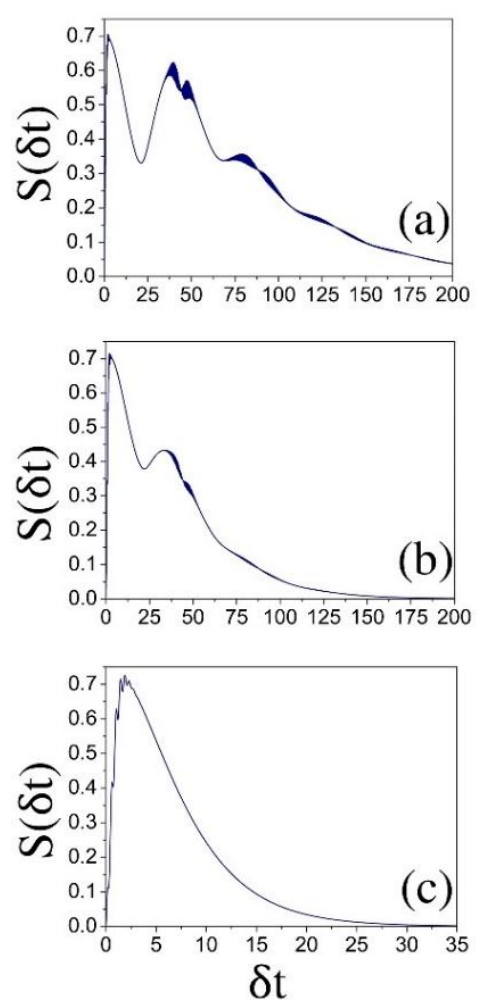

Fig. 3. Time evolution of the entropy for different values of the parameters $\beta$ : (a) $\beta=0.05 \delta$, (b) $\beta=0.09 \delta$, and (c) $\beta=0.50 \delta$, with $\alpha=7$, $\omega=\omega_{\mathrm{CPB}}=8.35 \delta$.

To substantiate the reliability of our TLR-CPB system, we use these following typical experimental values involving the system: coupling value of $\delta=100 \mathrm{MHz}$ and CPB frequency of $\omega_{\mathrm{CPB}}=835 \mathrm{MHz}$, see Ref. [25]. In Refs. [16], [26], we have that the TLR decoherence time is directly proportional to the lifetime and inversely proportional to the average excitation of TLR $\tau_{d} \sim \frac{\tau_{\text {life }}}{\langle\hat{n}\rangle_{L T R}}$. The lifetime of CPB is $\tau_{\text {life }}=200 \mu$ s [27] considering the very excited TLR, because the more excited the shorter the decoherence time $\langle\hat{n}\rangle=49$; thus, even for a relatively excited system our proposal has validity and still better it will become for a less excited system. We have a decoherence time at the TLR of $4 \mu \mathrm{s}$, the Figs. $2,3 \mathrm{a}$ and $3 \mathrm{~b}$, show a time of $\sim 2 \mu$ s below the decoherence time.
The authors would like to thank for the financial supports from Fundação de Amparo a Pesquisa do Estado de Goias (FAPEG), Coordenação de Aperfeiçoamento de Pessoal de Nível Superior (CAPES) and Conselho Nacional de Desenvolvimento Científico e Tecnológico (CNPQ). Xray data collecion was undertaken at the Department of Chemistry and Biochemistry of the University of Notre Dame. Research developed with support of the High Performance Computing Center at the UEG and Laboratório de Modelagem Molecular Aplicada e Simulação (LaMMAS).

\section{References}

[1] Z.-L. Xiang, S. Ashhab, J.Q. You, F. Nori, Rev. Mod. Phys. 85(2), 623 (2013).

[2] C. Jiang, Y.-S. Cui, H.-X. Liu, X.-W. Li, G.-B. Chen, Chinese Phys. B 24(5), 054206 (2015)

[3] T. Duty, Physics 3, 80 (2010).

[4] Q. Guo, L.-Y. Cheng, H.-F. Wang, S. Zhang, Chinese Phys. B 24(4), 040303 (2015)

[5] I. Buluta, S. Ashhab, F. Nori, Reports Prog. Phys. 74(10), 104401 (2011).

[6] J.J.L. Morton, B.W. Lovett, Annu. Rev. Condens. Matter Phys. 2(1), 189 (2011).

[7] C. Valverde, A.N. Castro, B. Baseia, Opt. Commun. 366, 301 (2016).

[8] R.-Y. Yan, H.-L. Wang, Z.-B. Feng, Int. J. Theor. Phys. 55(1), 251 (2016).

[9] J.A. Schreier et al., Phys. Rev. B 77(18), 180502 (2008).

[10] T.P. Purdy, D.W.C. Brooks, T. Botter, N. Brahms, Z.-Y. Ma, D.M. Stamper-Kurn, Phys. Rev. Lett. 105(13), 133602 (2010).

[11] P. Rabl, Phys. Rev. Lett. 107(6), 063601 (2011).

[12] J.-Q. Liao, F. Nori, Phys. Rev. A 88(2), 023853 (2013).

[13] J. van Wezel, T.H. Oosterkamp, Proc. R. Soc. A Math. Phys. Eng. Sci. 468(2137), 35 (2012).

[14] M.V. Gustafsson, T. Aref, A.F. Kockum, M.K. Ekstrom, G. Johansson, P. Delsing, Science 6206, 207 (2014).

[15] S.M. Girvin, M.H. Devoret, R.J. Schoelkopf, Phys. Scr. T137, 014012 (2009).

[16] C. Valverde, B. Baseia, Quantum Inf. Process. 12(5), 2019 (2013).

[17] T.J. Kippenberg, K.J. Vahala, Opt. Express 15(25), 17172 (2007).

[18] T.J. Kippenberg, K.J. Vahala, Science 321, 1172 (2008).

[19] I. Favero, K. Karrai, Nat. Photonics 3(4), 201 (2009).

[20] M. Aspelmeyer, T.J. Kippenberg, F. Marquardt, Rev. Mod. Phys. 86(4), 1391 (2014).

[21] R.J. Schoelkopf, S.M. Girvin, Nature 451, 664 (2008).

[22] A. Blais, R.-S. Huang, A. Wallraff, S.M. Girvin, R.J. Schoelkopf, Phys. Rev. A 69(6), 062320 (2004).

[23] Y.-L. Chen, Y.-F. Xiao, X. Zhou, X.-B. Zou, Z.-W. Zhou, G.-C. Guo, J. Phys. B At. Mol. Opt. Phys. 41(17), 175503 (2008).

[24] S.J.D. Phoenix, P.L. Knight, Phys. Rev. A 44(9), 6023 (1991).

[25] Z.-L. Xiang, X.-Y. Lü, T.-F. Li, J. Q. You, F. Nori, Phys. Rev. B 87(14), 144516 (2013).

[26] A.T. Avelar, B. Baseia, J. Opt. B Quantum Semiclassical Opt. 7(6), 19 (2005).

[27] Z. Kim et al., Phys. Rev. Lett. 106(12), 120501 (2011). 